\title{
Image Dependent Energy-Constrained Local Backlight Dimming
}

\author{
Burini, Nino; Nadernejad, Ehsan; Korhonen, Jari; Forchhammer, Søren; Wu, Xiaolin
}

Published in:

Proceedings of ICIP 2012

Link to article, DOI:

10.1109/ICIP.2012.6467480

Publication date:

2012

Link back to DTU Orbit

Citation (APA):

Burini, N., Nadernejad, E., Korhonen, J., Forchhammer, S., \& Wu, X. (2012). Image Dependent EnergyConstrained Local Backlight Dimming. In Proceedings of ICIP 2012 (pp. 2797-2800). IEEE. International Conference on Image Processing. Proceedings https://doi.org/10.1109/ICIP.2012.6467480

\section{General rights}

Copyright and moral rights for the publications made accessible in the public portal are retained by the authors and/or other copyright owners and it is a condition of accessing publications that users recognise and abide by the legal requirements associated with these rights.

- Users may download and print one copy of any publication from the public portal for the purpose of private study or research.

- You may not further distribute the material or use it for any profit-making activity or commercial gain

- You may freely distribute the URL identifying the publication in the public portal 


\title{
IMAGE DEPENDENT ENERGY-CONSTRAINED LOCAL BACKLIGHT DIMMING
}

\author{
Nino Burini ${ }^{1}$, Ehsan Nadernejad ${ }^{1}$, Jari Korhonen ${ }^{1}$, Søren Forchhammer ${ }^{1}$, and Xiaolin $W u^{2}$ \\ ${ }^{1}$ DTU Fotonik, Technical University of Denmark, Kgs. Lyngby, Denmark \\ ${ }^{2}$ McMaster University, Hamilton, Canada
}

\begin{abstract}
In this work, we consider and propose two extensions to an optimization-based image dependent backlight dimming algorithm. The first extension introduces error weighting based on human perception of luminance, aiming to improve the perceived image quality; the second extension adds an adjustable term for power consumption to the cost function, allowing flexible power management. Experimental results show that the proposed solution can achieve better results than other algorithms at several power consumption levels.
\end{abstract}

Index Terms - Liquid crystal display, Local backlight dimming, Optimization, Image quality, Power management

\section{INTRODUCTION}

Liquid Crystal Displays (LCDs) are used on a wide variety of devices, including TV sets and computer monitors. Liquid crystals do not emit light; the light is generated by the backlight instead, then modulated by the crystals, which - thanks to proper color filters - can render a large gamut. Today Light Emitting Diodes (LEDs) are common light sources for LCD backlight, due to attractive characteristics like flexibility in use and growing efficiency [1].

In LCDs a large amount of light is dissipated and only a small fraction, down to less than $10 \%$, reaches the viewer [2]. For this reason, the backlight must emit intense light, which makes it the most power consuming component in the display. LCD are also affected by light leakage: LC cannot fully block light when reproducing black or very dark pixels, making them look slightly grayish. Light leakage is the reason for the limited contrast ratio in LCDs, and it is especially visible from wide viewing angles.

Leakage and power consumption can be reduced by dimming the backlight, and instead increase the transmittance of the LC to compensate the lower light output. This allows to decrease light emission, while rendering the same image with little or no distortion. LED technology has eased the implementation of independently controllable segments of local backlights, as opposed to global backlight, where the luminance is uniform across the whole display. In this paper, the terms "backlight segments" and LED are used interchangeably. Depending on the image content, local backlight dimming gives significant opportunities for power saving and contrast improvement. However, aggressive dimming can cause clipping: when the LC cannot compensate the reduction of light intensity, pixels appear less bright then they should. In this work, we refer to pixels affected by clipping as "clipped" pixels.

Several backlight dimming algorithms exist; many of them focus on reducing power consumption while keeping an acceptable quality [3][4][5]. In [6], backlight dimming is modeled as an image optimization problem. The optimum backlight illumination defined by a cost function and by the input image is found; the result is a compromise between clipping and leakage. The focus is on increasing contrast and image quality and taking power savings as a positive side effect. We propose to extend this algorithm by introducing weighting of errors based on luminance perception by the Human Visual System (HVS), and by adding an adjustable term for power consumption to the cost function.

The rest of this paper is organized as follows. Section 2 presents the fundamentals of modeling LCD with backlight dimming systems. In Section 3, the proposed extensions are illustrated. Section 4 shows the experimental results and, finally, the concluding remarks are given in Section 5.

\section{MODELING BACKLIGHT DIMMING SYSTEMS}

Figure 1 illustrates a generic LCD display with local backlight dimming. The backlight dimming algorithm takes the digital image to be displayed as input, and produces the intensities for each backlight segment and the transmittance of LC pixels as output. The observed luminance $L$ at pixel $(i, j)$ can be calculated as the product of the backlight intensity $B$ behind the pixel and the transmittance $T$ of the pixels itself:

$$
L(i, j)=B(i, j) T(i, j)
$$

where $B$ and $T$ are normalized to the interval $[0,1]$.

The digital input image is typically presented in an ideally perceptually uniform format, i.e. similar intervals of pixel values represent similar differences in perceived intensity over the whole range. Since the relationship between physical luminance and perceived brightness is not linear, practical displays usually perform gamma correction when the input image is converted to LC transmittance. Gamma correction is good for conventional displays with relatively low peak luminance (up to about $100 \mathrm{~cd} / \mathrm{m}^{2}$ ), but more accurate functions have been proposed for perceptual linearization of the input signal in brighter displays [7]. 


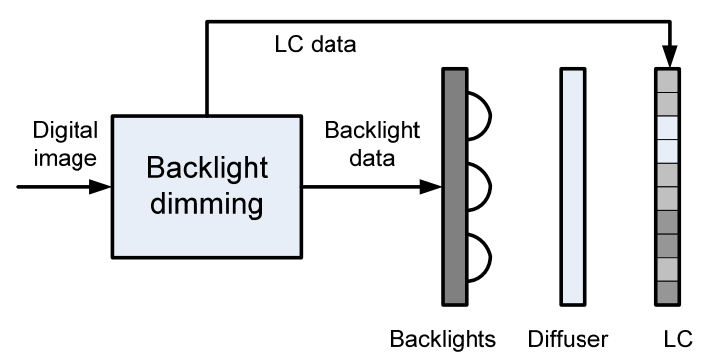

Figure 1. Generic local backlight dimming system.

Since HVS is very sensitive to contrast changes at low physical luminance levels, light leakage becomes a problem in dark parts of images. The impact of leakage on the observed luminance can be included by modifying Eq. (1):

$$
L(i, j)=B(i, j) T(i, j)+\varepsilon(i, j) B(i, j)(1-T(i, j)),
$$

where $\varepsilon(i, j)$ is a leakage factor representing the amount of light leaking through a black LC pixel. Leakage and $\varepsilon(i, j)$ depend on pixel position and viewing angle; however, in this work, we assume a constant $\varepsilon$ across the display.

In practical LCDs, light from independent backlight segments is diffused and mixed on the diffuser plate between backlight units and LC layer. In order to compute the backlight intensity at each pixel, we need to know how the light is distributed around the center of a light source. It is assumed that the distribution follows a Point Spread Function (PSF) specific to the display, multiplied by the normalized luminance of the backlight unit. Each pixel position is usually influenced by several backlight units, and the total luminance can be computed simply by summing the individual contributions. The PSF is sometimes approximated by a two-dimensional Gaussian function, but for more accurate results, the PSF should be determined experimentally for each display. The backlight intensity at pixel $(i, j)$ can be computed as follows:

$$
B(i, j)=\sum_{k=1, N} B_{k} h_{k}(i, j)
$$

where $B_{k}$ is the intensity of backlight unit $k$, and $h_{k}$ is the PSF expressing the relative amount of light from backlight $k$ reaching pixel $(i, j)$. Since the backlight dimming algorithm may have dimmed the light intensity significantly in the regions dominated by dark pixels, the so-called "brightness compensation" is performed to restore the original target luminance. This is done by increasing the LC transmittance $T$ so that the target $L$ is reached.

Using the Eqs. (2) and (3), it is possible to compute the observed luminance of each pixel, or in case of color display, the luminance of R, G and B subpixels, out of the intensities of individual backlights and LC transmittance values. The modeled luminance can be converted back to uniform scale by performing inverse Gamma correction and rescaling normalized values to 8-bit integers. Then, it is possible to compare the modeled image on the display against the original input using traditional measures, such as
Mean Squared Error (MSE), Peak Signal-to-Noise Ratio (PSNR), or any other image quality indicator.

\section{PROPOSED ALGORITHM}

An algorithm minimizing the error was presented in [6]. In this work, we propose two extensions: one extension is image dependent individual weighting of the pixel error, based on perceptual aspects; the other extension is the introduction of a term representing power consumption in the cost function.

As in [6], the algorithm models backlight dimming as an optimization problem. The distortion to be minimized is the difference between an ideal output $y$ and the actual output $x$ rendered on the screen, which is determined by the backlight $b$ as derived from Eq. (3), the compensated pixel values $a$ and the leakage factor $\varepsilon$; the constraints include the values of pixel transmittance and LED intensity. The other term is power $p$ multiplied by a weighting parameter $q$. Optimization can be done in $\ell_{1}$ or $\ell_{2}$; the experiments in this work used $\ell_{1}$ as it allows to solve the problem using linear programming. For more details, the reader may consult [6]. The formulation of the problem, with the proposed extensions, is given as:

$$
\begin{aligned}
& \operatorname{minimize}\|(y-x) \circ w\|+q \times p \\
& \text { subject to } x=a \circ b \\
& b=H r \\
& \varepsilon \leq a \leq 1 \\
& 0 \leq r \leq 1
\end{aligned}
$$

where the constraint $b=H r$ corresponds to Eq. (3) and the term $(y-x)$ is multiplied point-wise by a weighting matrix $w$. The original formulation of the optimization problem [6] can be obtained again by setting $w$ to a matrix of ones and by setting $q$ to zero.

The original formulation solves the backlight problem in the physical domain, meaning that it deals with luminance and pixel transmittance to find the light output that matches the target as closely as possible. However, the HVS has a nonlinear response to luminance, and the perceived luminance $L_{P}$ is a function of the physical luminance $L$ : $L_{P}=f(L)$. The function $f$ can be modeled in several ways. Without loss of generality, we can approximate the HVS response to luminance with an inverse gamma function:

$$
f(L)=L^{1 / \gamma}
$$

A typical value for $\gamma$ is 2.2 [8]. Due to the nonlinearity of HVS, a small change of a low luminance level will be more noticeable than the same change at a higher luminance level. This implies that, given the same absolute error in physical luminance, leakage is more noticeable than clipping. For this reason, the optimal "physical" solution to the backlight problem might not be the optimal perceived solution. We propose to modify the cost function by adding a weighting matrix $w$ that assigns a weight to the error, depending on the target luminance of the pixel in question.

$$
w_{i, j}=f^{\prime}\left(y_{i, j}\right)
$$


In this work, the weight is calculated from the gamma curve at the normalized target luminance value. Since the slope of the curve is steeper at low luminance, errors in the dark pixels will be weighted more than those in the high luminance area, providing a better match to the perception model. The concept of the weighting matrix $w$ can be extended to include any kind of image dependent weighting applied to the error which is to be minimized.

The second extension proposed adds a power term to the cost function. The optimization-based algorithm aims to achieve the best possible image quality and considers power savings as a positive side effect. However, lower image quality might be acceptable in exchange of lower power consumption, and it is interesting to study the tradeoff between the two factors. The power term in the cost function includes the mean of the normalized LED intensities (Eq. (7)), which estimates power consumption:

$$
p=\frac{\sum_{k=1}^{N} B_{k}}{N}
$$

\section{EXPERIMENTAL RESULTS}

The experiments conducted here aim at evaluating the performance of the proposed algorithm with different power weights in the cost function and at different image resolutions. The first experiment compares the impact of image downscaling for the proposed algorithm on two simulated displays. The second experiment compares the proposed algorithm against other algorithms as the power weight factor and the image downscaling factor vary.

The proposed algorithm has been compared with other algorithms described below. In the Max and Avg algorithm [9], the intensity of each backlight segment is set to the maximum or average value of the segment pixels; only the Max algorithm has been considered for the experiment. The other algorithms cited here are referred to by the name of the first author of the respective publication. In Cho e.a. [3], the algorithm analyzes each segment and sets the backlight luminance to the sum of the average value of the segment pixels and a correction value, which depends on the average and the maximum value of the segment. In Nam [4], the backlight luminance can be set locally or globally depending on the type of image. LED intensities for global and local cases are set according to the mean of the maximum and average values of the total image and segment, respectively. Finally, in Albrecht e.a. [5] a clipper-free algorithm consisting of three steps is proposed: the first step sets lower bounds for each backlight segment, depending on the image content and on the PSF; the optional second step is iterative: for each iteration, it increases the luminance of one LED until there are no clipped pixels, or another ending condition is met; the third step scans the pixels of each segment in a specific order and adjusts the LED values to make sure that every pixel receives enough backlight.

Two screen models have been used for the experiments. The first one is a simulated device with 221 backlight segments places in a grid of 13 rows and 17 columns; the
PSF is given by a Gaussian function. The second one is modeled on a 47" local backlight dimming Full HD screen manufactured by SIM2 [10]. The screen has 2202 backlight segments placed in a hexagonal grid behind the LC layer; the PSF has been provided by the manufacturer. For our simulations, leakage has been modeled with an $\varepsilon$ value of 0.001 for both screens.

Eight images were used in the experiments. The set of chosen images includes several image types like synthetic graphics, natural images and compressed pictures. For the simplicity of error analysis, all the images are in grayscale.

The target luminance $y$ is calculated from the sRGB input image $I$ as the CIE 1931 luminance component $Y(8)$. The complexity of the proposed algorithm grows very quickly with the image size; e.g., solving the optimization problem for a Full HD image on the SIM2 screen implies millions of variables and constraints. It is possible to reduce the complexity by downscaling the input images. Depending on the experiment, the input images have been downscaled by a factor varying between 1 (original size) and 10 using bicubic interpolation. The PSF of the screens have been downscaled accordingly. Even if the backlight is calculated from a downscaled version of the input image, the resulting $B_{k}$ values are applied at full resolution; after brightness compensation, distortion is measured in PSNR between the normalized input image $I$ and the output of the algorithm $x$ after applying Eq. (5) to each $L(i, j)$ obtained from Eq. (2) at full resolution:

$$
P S N R=10 * \log _{10}\left(\frac{N}{\sum(I-f(x))^{2}}\right),
$$

where $N$ is the number of pixels in $I$ and $x$. Figure 2 shows that both the PSNR and power consumption of the solution obtained from the proposed algorithm increase as the downscaling factor decreases; the factor was varied between 1 and 10 for the screen with Gaussian PSF, and between 4 and 10 for the SIM2 screen.

Figure 3 shows the comparison between the proposed method and the other algorithms presented earlier. The results have been calculated for the optimal backlight with 13 positive values of the power weight factor $q$ (Eq. 4$)$. The resulting curve highlights the tradeoff between power consumption and PSNR. For a given power level, the proposed solution can achieve a better outcome than the other algorithms: the PSNR for the proposed algorithm is approximately $3 \mathrm{db}$ higher than the Max, Nam, Cho and Albrecht solutions at the power level of each of these; with the same PSNR, the proposed algorithm reduces power consumption by 0.2 compared to Albrecht. It is interesting to notice that for low $q$ values (right part of the curve in the figure) a modest increase of error is associated with a significant reduction in power consumption.

Table 1 shows the relative contributions to MSE from leaking and clipped pixels for all algorithms. Three $q$ values are considered for the proposed method. Using the notation of problem (4), pixel $(i, j)$ is defined as leaking if $a=0$ and $x(i, j)>y(i, j)$ and it is defined as clipped if $a=1$ and 


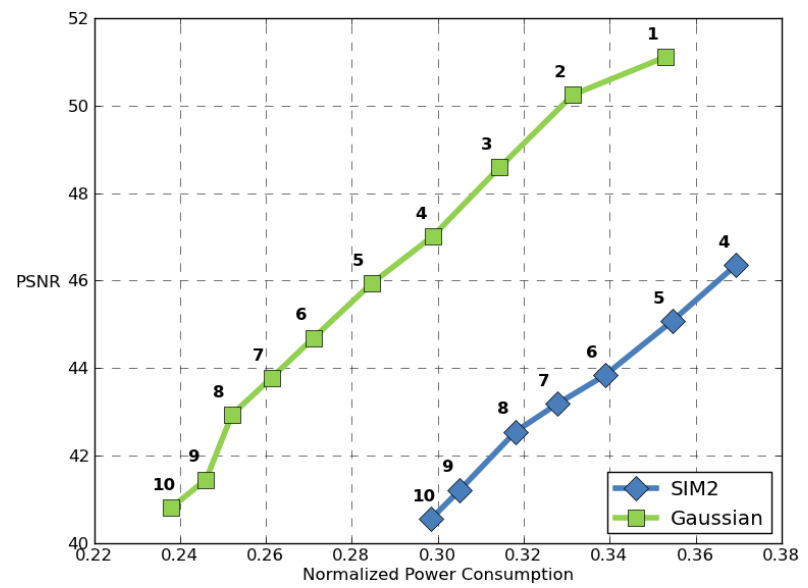

Figure 2. Impact of downscaling factor (above the dots) on PSNR and power consumption.

$x(i, j)<y(i, j)$. The error in Max, Nam and Cho is almost completely caused by clipping, as they fail to provide enough light to many pixels. The error in the Albrecht algorithm is mostly due to leakage instead; this is expected, since the algorithm is clipper free. There is a small clipping error caused by the algorithm being run on a downscaled image, causing clipping in the full resolution image. As for the proposed algorithm, the impact of leakage and clipping depends on the power factor $q$. Leakage is dominant when $q$ is equal to zero, but clipping pixels become more important as $q$ increases. This is easily explained by the fact that a higher $q$ favors low LED intensities, resulting in more pixels being clipped.

\section{CONCLUSIONS}

We have presented two extensions for an optimizationbased image dependent backlight dimming algorithm [6] to provide a tradeoff between perceived distortion and power. The proposed solution can achieve a PSNR improvement of about $3 \mathrm{db}$ compared to other algorithms at the level of power consumed by these. Downscaling the input image prior to optimization reduces complexity and power consumption, although at the price of a higher mean square error. Leakage is the main cause for errors at high power levels, while clipping becomes dominant at low levels. Future work will include reducing the complexity of the algorithm, and investigation of the perceptual weighting of errors.

Table 1: average absolute contribution of leaking and clipped pixels on MSE $\left(\cdot 10^{-5}\right)$ and related percentage.

\begin{tabular}{|l|c|r|c|r|}
\hline Algorithm & \multicolumn{2}{|c|}{ Leakage } & \multicolumn{2}{c|}{ Clipping } \\
\hline Max & 2.49 & $4.92 \%$ & 48.1 & $95.04 \%$ \\
\hline Nam & 0.84 & $0.42 \%$ & 197.0 & $99.57 \%$ \\
\hline Cho & 1.91 & $2.19 \%$ & 85.2 & $97.79 \%$ \\
\hline Albrecht & 25.1 & $97.73 \%$ & 0.54 & $2.09 \%$ \\
\hline Proposed q=0 & 5.5 & $65.30 \%$ & 2.85 & $33.82 \%$ \\
\hline Proposed q=1468 & 2.99 & $17.61 \%$ & 13.9 & $82.24 \%$ \\
\hline Proposed q=19818 & 0.88 & $0.66 \%$ & 13.1 & $99.33 \%$ \\
\hline
\end{tabular}

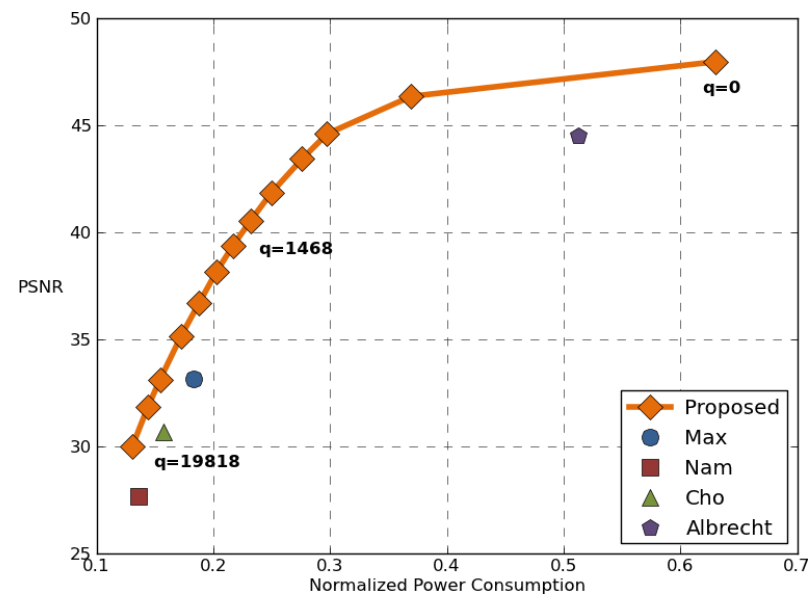

Figure 3. Comparison of the proposed solution against other algorithms at various power weights $q$ (Eq. 4).

\section{REFERENCES}

[1] M. Anandan, "Progress of LED backlights for LCDs," Journal of the SID, vol. 16, no. 2, pp. 287-310, 2008.

[2] H. Groot Hulze and P. de Greef, "Power Savings by Local Dimming on a LCD Panel with Side Lit Backlight," SID Symposium Digest of Technical Papers, vol. 40, no. 1, pp. 749-752, 2009.

[3] H. Cho and O. K. Kwon, "A backlight dimming algorithm for low power and high image quality LCD applications," Consumer Electronics, IEEE Transactions on, vol. 55, no. 2, pp. 839-844, 2009.

[4] H. Nam, "Low power active dimming liquid crystal display with high resolution backlight," Electronics Letters, vol. 47, no. 9, p. 538, 2011.

[5] M. Albrecht, A. Karrenbauer, T. Jung, and C. Xu, "Sorted Sector Covering Combined with Image Condensation-An Efficient Method for Local Dimming of Direct-Lit and Edge-Lit LCDs," IEICE Transactions on Electronics, vol. 93, no. 11, pp. 1556-1563, 2010.

[6] X. Shu, X. Wu, and S. Forchhammer, "Optimal local dimming for LED-backlit LCD displays via linear programming," in Proceedings of SPIE-IS\&T Electronic Imaging, 2012, p. 830517.

[7] J. Korhonen, N. Burini, S. Forchhammer, and J. M. Pedersen, "Modeling LCD displays with local backlight dimming for image quality assessment," in Proceedings of SPIE-IS\&T Electronic Imaging, 2011, vol. 7866, pp. 786607-786607-9.

[8] M. Stokes, M. Anderson, S. Chandrasekar, and R. Motta, "A Standard Default Color Space for the Internet - sRGB," Microsoft and Hewlett. pp. 238-245, 1996.

[9] Funamoto T, Kobayashi T, and Murao T, "High-PictureQuality Technique for LCD televisions: LCD-AI," in Proc Int Disp Workshops, 2001, pp. 1157-1158.

[10] http://www.sim2.com/HDR/ 